\title{
Interdomain communication between weak structural elements within a disease-related human tRNA
}

\author{
Marc D. Roy, Lisa M. Wittenhagen, Brian Vozzella, and Shana O. Kelley* \\ Boston College, Eugene F. Merkert Chemistry Center, Chestnut Hill, MA 02467

\section{Supporting information}

Figure S1. Enzymatic probing of WT and anticodon stem mutants of hs mt tRNA ${ }^{\mathrm{Leu}(\mathrm{UUR})} \cdot 5^{,}-{ }^{32} \mathrm{P}$-labeled tRNAs were subjected to enzymatic cleavage under non-denaturing conditions $\left(40 \mathrm{mM} \mathrm{NaCl}, 10 \mathrm{mM} \mathrm{MgCl}_{2}, 1\right.$ $\mathrm{mM}$ spermine). Samples were analyzed using 12\% denaturing PAGE. Lanes numbered 1, 2, and 3 represent results for WT tRNA ${ }^{\text {Leu(UUR) }}$ and the U40C and U40C/A30G mutants, respectively. Samples were run alongside untreated control tRNA (C). Lanes labeled S1, T2 and V1 represent non-denaturing cleavage results for each enzymatic probe. Also shown is a denatured RNase T1 ladder $(\mathrm{G})$ and an alkaline ladder (A). Numbers to the left of the gel indicate the guanosine position in the RNase T1 ladder of WT tRNA ${ }^{\text {Leu(UUR) }}$.

Figure S2. Structural probing of $5{ }^{3}-{ }^{32} \mathrm{P}$-labeled WT and D stem mutants of hs $\mathrm{mt}$ tRNA $^{\text {Leu(UUR) }}$ using nuclease S1, and RNase T2 under non-denaturing conditions ( $40 \mathrm{mM} \mathrm{NaCl}, 10 \mathrm{mM} \mathrm{MgCl} 2,1 \mathrm{mM}$ spermine). Samples were analyzed using 20\% denaturing PAGE, and were run alongside untreated 
control tRNAs (C), a denatured RNase T1 ladder (G), and an alkaline ladder (A). Numbered lanes 1-5 represent results for each of the analyzed tRNAs (1:WT, 2:DTP, 3:A12G, 4:U22C, 5:C23U). Numbers to the left of the gel indicate the guanosine position in the RNase T1 ladder of WT tRNA ${ }^{\mathrm{Leu}(\mathrm{UUR})}$.

Figure S3. Native PAGE of WT (UUR), C23U, DTP, WT (CUN), A12G, and U22C hs $m t$ tRNA ${ }^{\text {Leu }}$ (lanes 1-6, respectively). The hs $m t$ tRNA ${ }^{\text {Leu(CUN) }}$ migrates faster as it contains 74 nucleotides whereas hs $m t$ tRNA ${ }^{\text {Leu(UUR) }}$ contains 78 nucleotides. The gel was visualized using ethidium bromide staining.

Figure S4. (A) Thermal denaturation profiles for $0.38 \mathrm{uM}$ WT(UUR) $(\Delta)$, U22C $(\diamond)$, DTP $(\square)$, and WT(CUN) (O) hs mt tRNA ${ }^{\text {Leu }}$. (B) First derivative curves of the thermal denaturation curves for the same samples as indicated above. The $\mathrm{T}_{\mathrm{m}}$ for WT (UUR), U22C, DTP, and WT (CUN) hs mt $\mathrm{tRNA}^{\mathrm{Leu}}$ were determined from the maxima of each curve and were 54.3, $54,55.2$, and $58.2^{\circ} \mathrm{C}$ respectively. 


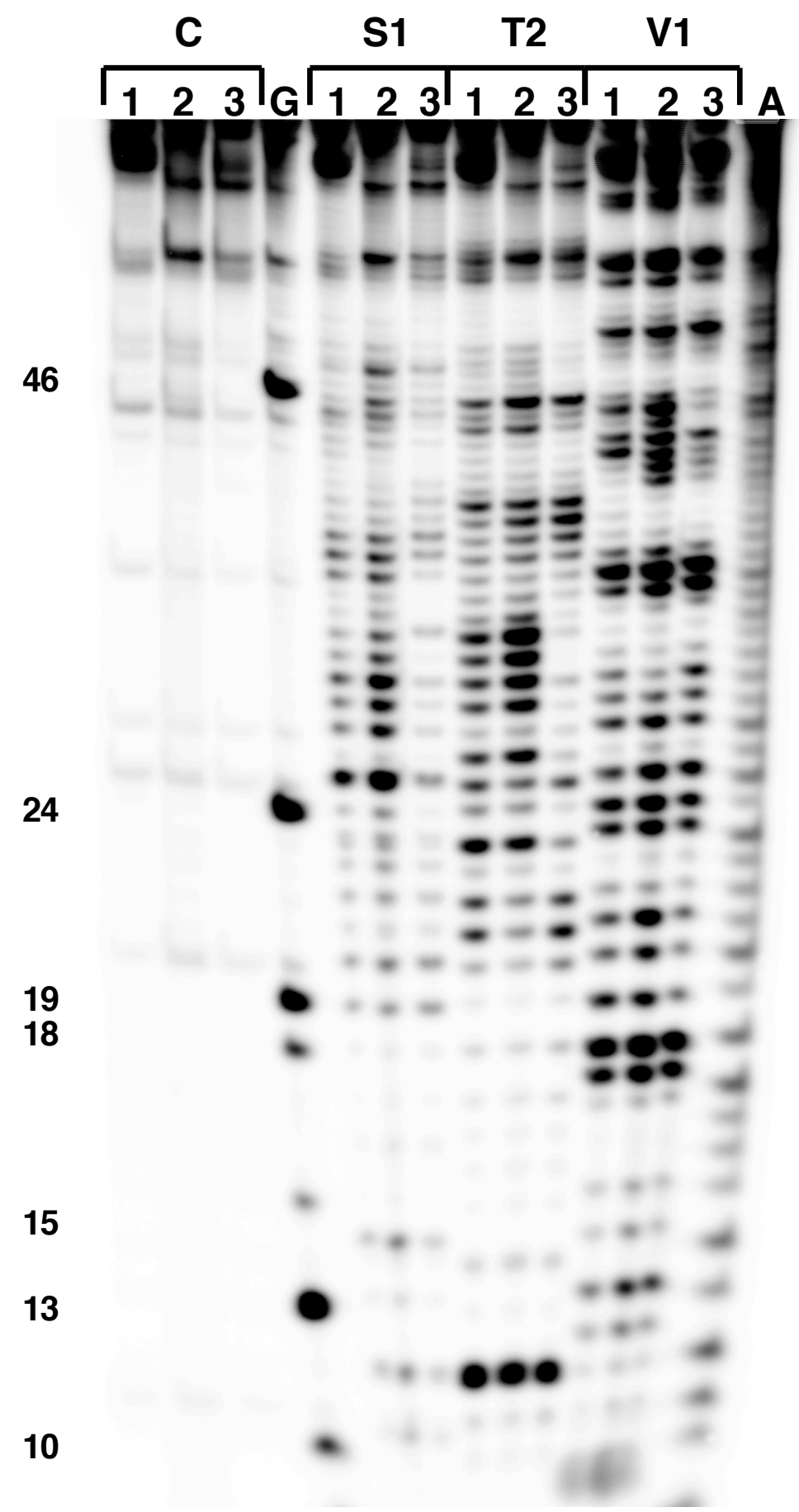

Figure S1 


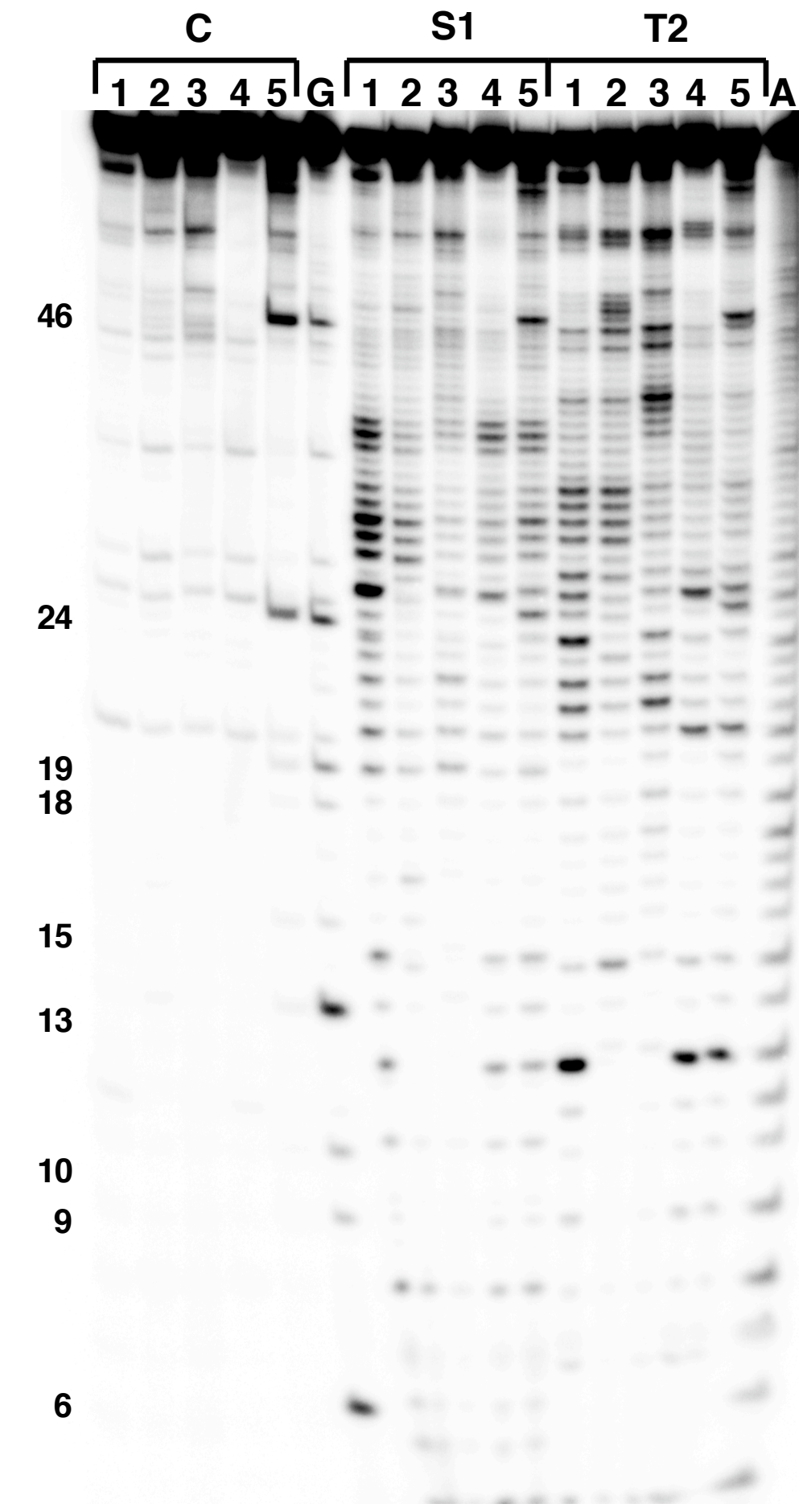

Figure S2 


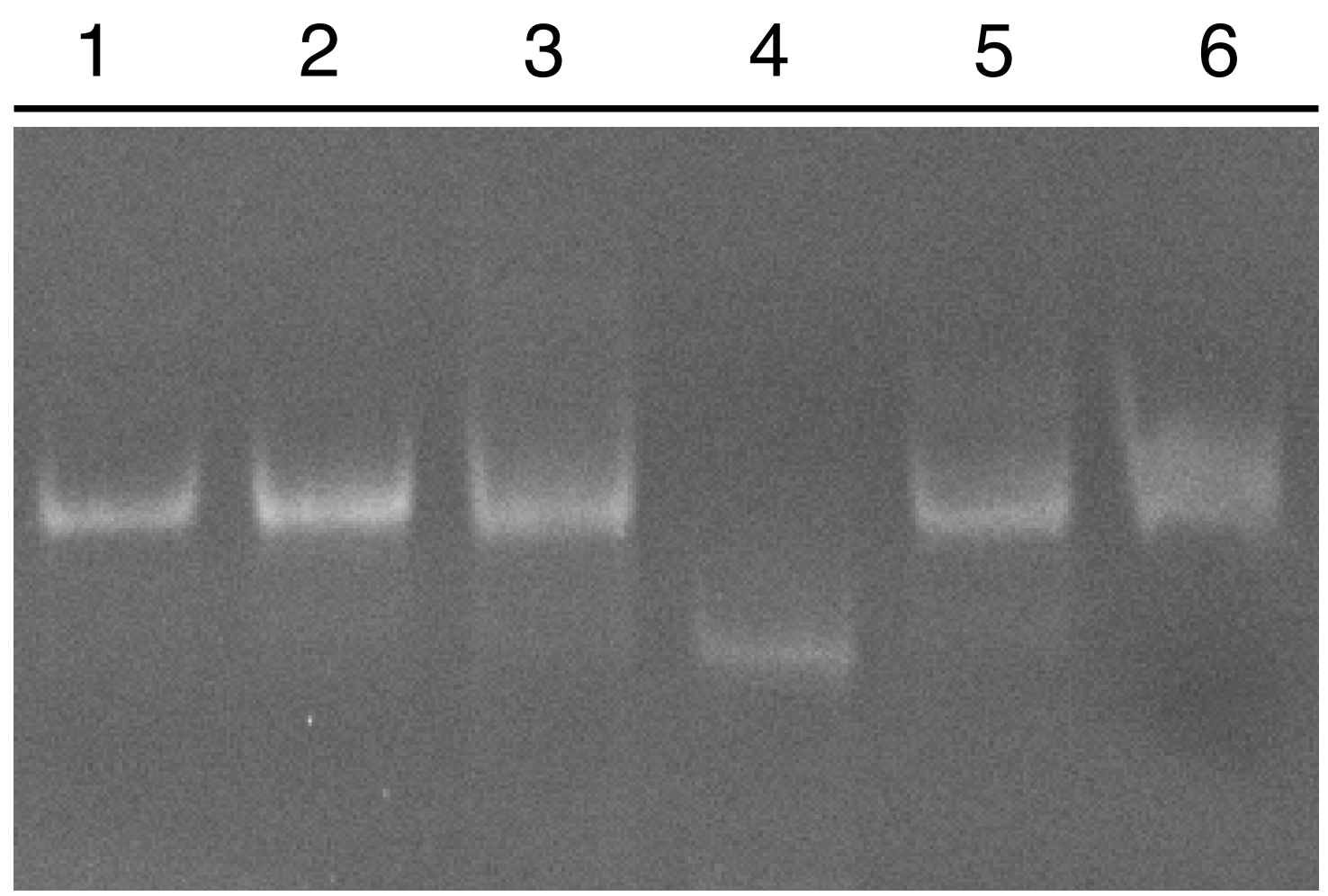

Figure S3 


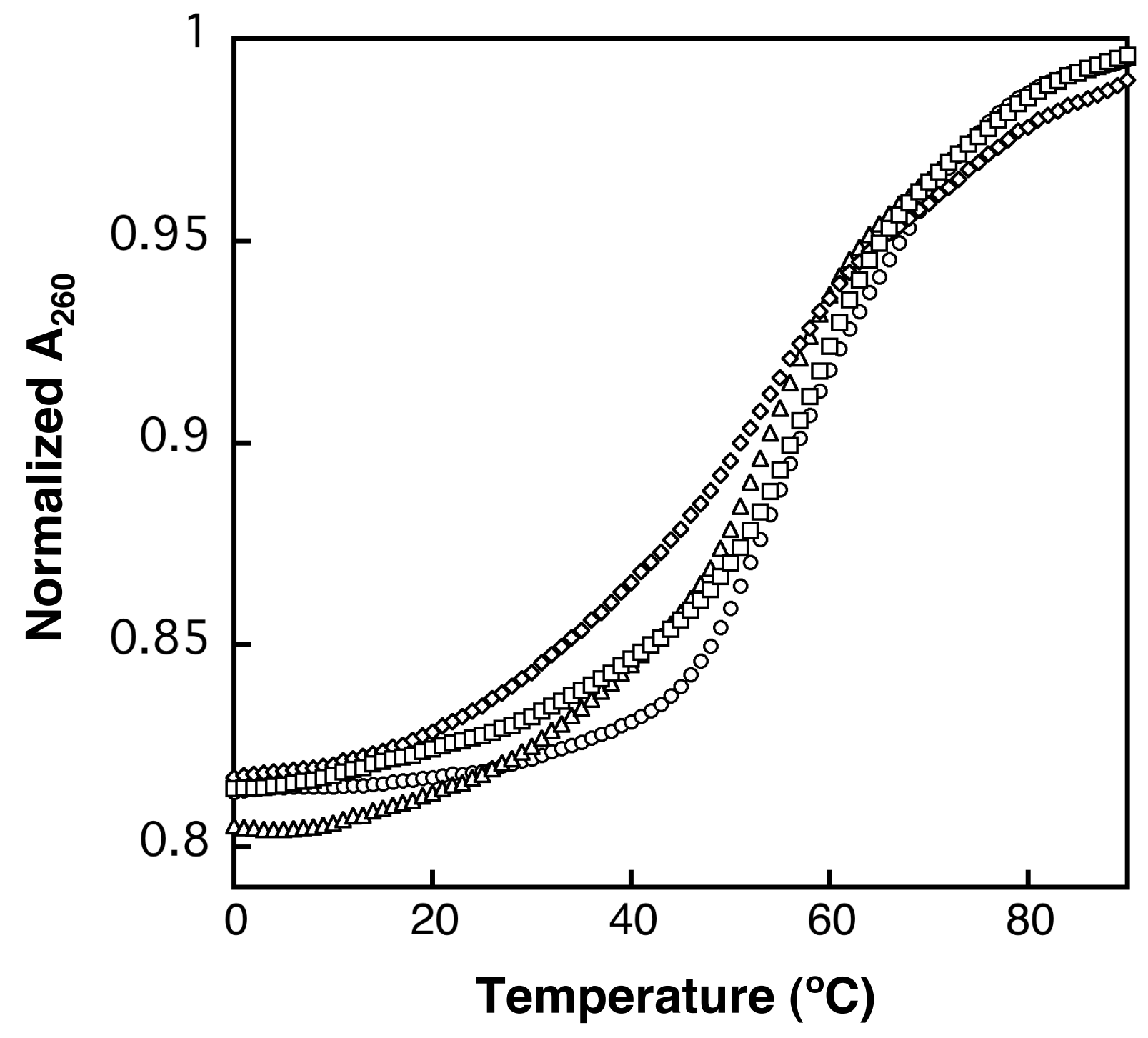

Figure S4A 


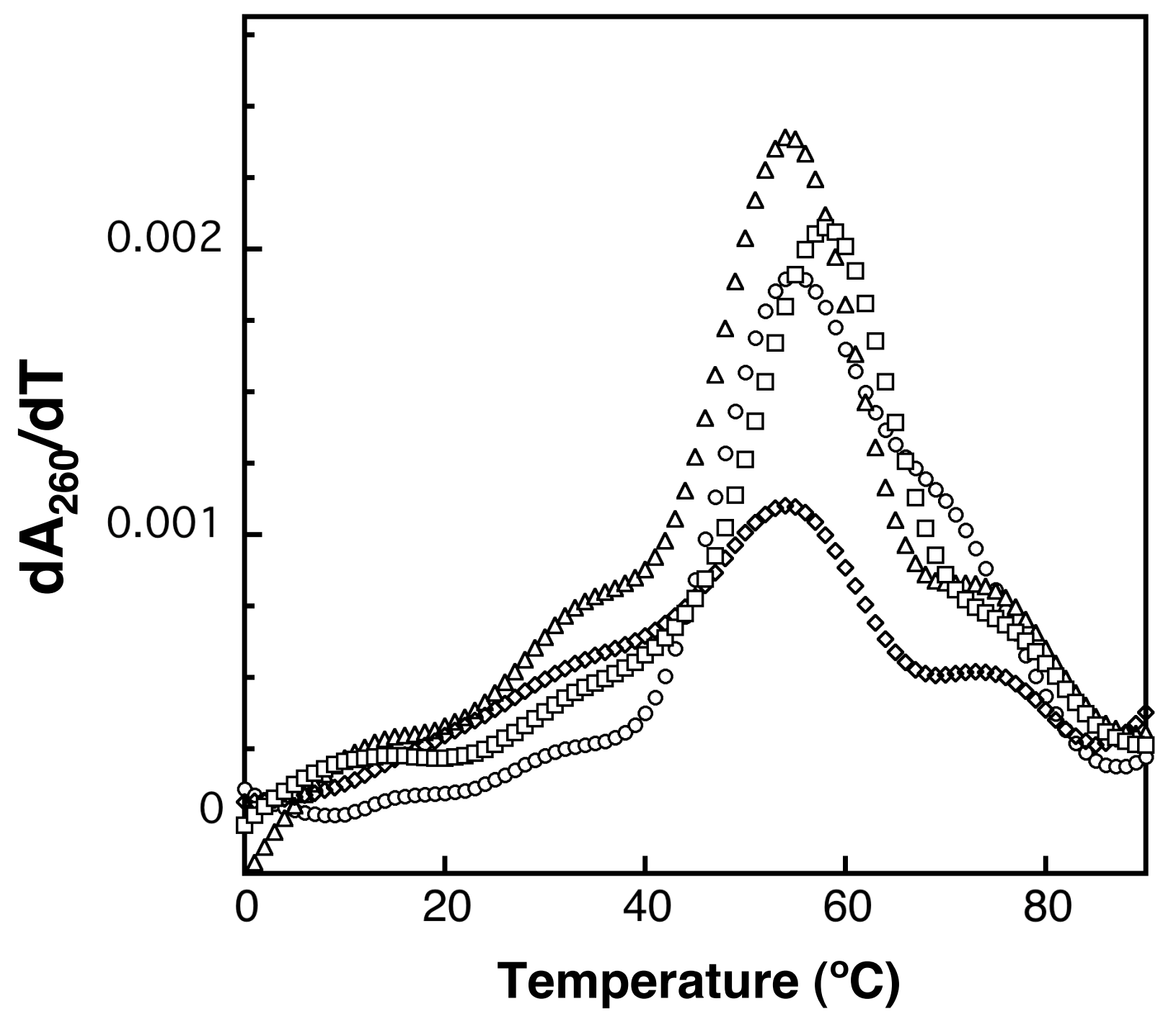

Figure S4B 